\title{
A HYBRID SIMULATION FRAMEWORK FOR THE NEWSVENDOR PROBLEM WITH ADVERTISING AND VIRAL MARKETING
}

\author{
Ashkan Negahban \\ Department of Industrial and Systems Engineering \\ Auburn University \\ Auburn, AL 36849, USA
}

\begin{abstract}
The newsvendor problem is known as one the classical problems in the inventory management context which has received a great deal of attention during the past few decades. In this paper, a two-level simulation-based framework is proposed, where in the first level, agent-based simulation is developed to model the effect of advertising intensity and word-of-mouth on the demand in order to estimate the demand distribution under various levels of advertising intensity. The results from the agent-based model are then plugged into a Monte Carlo simulation model in order to make the final decision on the optimal advertising intensity and economical order quantity with the objective to maximize the expected profit. The proposed approach is then applied to a hypothetical newsvendor problem to illustrate its applicability as a decision support tool for solving real-world newsvendor problems.
\end{abstract}

\section{INTRODUCTION}

The original newsvendor problem (NVP) involves maximizing the expected profit from a single-period procurement decision on the order quantity of a single product with stochastic demand. At the end of the sales period, the remaining inventory units will be sold at a scrap price lower than the initial unit purchase cost. Ordering too few units, on the other hand, will result in losing potential customers, i.e. lost sales. Therefore, overstocking and understocking will both result in reduced overall profit. The insights stemming from this classical problem have significant implications in a wide spectrum of domains including manufacturing systems, airlines, fashion goods, retail stores, and distribution centers. During the past decade, as product customization has intensified resulting in shorter product life cycles and higher levels of demand uncertainty, more and more products are acquiring the attributes of seasonal or fashion goods such as those encountered in the newsvendor problem (Petruzzi and Dada 1999), which further necessitates development of more efficient tools to solve this problem.

There is a vast literature on the analysis of the newsvendor problem. However, in most of these studies, demand or market parameters are taken to be exogenous and deterministically known or given (Petruzzi and Dada 1999). Although very few studies consider the effect of advertising by modeling the mean demand as a function of advertising or marketing efforts (Qin et al. 2011; Khouja 1999), the role of individual consumers and their interactions through word-of-mouth, also known as viral marketing, have been completely ignored. This paper aims to address the above deficiencies in the existing literature by proposing a novel hybrid approach integrating Agent-Based Modeling and Simulation (ABMS) and Monte Carlo Simulation (MCS). In the first level of the framework, the distribution of future demand is estimated from the results of the ABMS analysis where demand emerges as a result of advertising, word-of-mouth, and individual consumer decision making process. In the second level, a Monte Carlo simulation model of the newsvendor problem is used to characterize the expected profit for various levels of initial advertising and determine the the economical order quantity that maximizes the profit. 


\section{Negahban}

The remainder of the paper is organized as follows. A review of the related literature is provided in Section 2. Section 3 presents the general structure of the proposed framework as well as technical details regarding different components of the agent-based and Monte Carlo simulation models. The implementation of the hybrid ABMS-MCS in a hypothetical newsvendor problem is illustrated in Section 4. Finally, conclusions and future research opportunities are provided in Section 5.

\section{LITERATURE REVIEW}

Early work in the field typically involves extensions to the classical single-period model to account for the following factors. For the sake of conciseness, a short description of each category is presented along with a sample reference. A complete list of these studies is provided in Khouja (1999).

- Objectives (Lau 1980): The objective in the classical NVP is to maximize the expected profit. However, other objectives including effectiveness criterion and utility functions are also studied.

- Supplier pricing policies (Jucker and Rosenblatt 1985): A fixed initial cost per unit quoted by the supplier is the standard assumption in NVP. However, suppliers tend to quote quantity discount schemes to generate larger order quantities from the buyer. The impact of alternative supplier discounting schemes have been studied in the literature.

- Multi-product models (Nahmias and Schmidt 1984): This category includes various extensions involving decision making on the economical order quantity for multiple products.

- Multi-echelon systems (Eynan and Rosenblatt 1995): The NVP in the context of stock levels for multi-stage systems such as assembly-to-order and assembly-in-advance systems is also studied.

- Multi-period models (Murray and Silver 1966): The idea behind these models is that there are multiple opportunities to purchase the item at pre-specified points in time where the purchase cost depends on the purchase time.

More recently, several other extensions to the original problem have been proposed. For a comprehensive review of these studies, see Qin et al. (2011).

- Price-dependent demand (Petruzzi and Dada 1999): These studies assume that customer demand and price are interrelated.

- Advertising-sensitive demand (Kraiselburd, Narayanan, and Raman 2004): Under this category, customer demand can be influenced by advertising and marketing efforts by the firm.

- Stock-dependent demand (Urban 2002): In the models in this category, the demand is a function of the order quantity.

- Risked-based analysis (Eeckhoudt, Gollier, and Schlesinger 1995): In classical NVP, the firm is risk-neutral and the only objective is to maximize the value of the expected profit. Under this category, the risk associated with different order quantities and firm's risk profile are also considered.

The literature review reveals two major gaps in the current literature. First, in most of the studies, the demand is assumed to be exogenous. Although, advertising-sensitive demand and supplier pricing policies are addressed separately, their impact on the economical order quantity has not been studied simultaneously, perhaps because it makes the problem too complicated to be solved analytically. Secondly, the impact of word-of-mouth and viral marketing has not been addressed in the literature since aggregate analytical models simply are not capable of capturing the interactions between customers at the individual level. This paper contributes to the existing literature in two significant ways: (1) to the best of the author's knowledge, this is the first study that explicitly captures word-of-mouth and its impact on the dynamics of the newsvendor problem; and (2) the paper proposes a novel approach based on agent-based and Monte Carlo simulations that allows for investigating the impact of initial advertising, viral marketing, supplier pricing policies, and risk-based analysis all at the same time. 


\section{Negahban}

\section{THE PROPOSED HYBRID SIMULATION FRAMEWORK}

The proposed framework consists of two main levels. In the first level, agent-based simulation is employed to model different dynamics of the market place including advertising, consumer decision making, and viral marketing. After verification and validation, the model is used to estimate the demand distribution under various levels of advertising intensity. The results of the ABMS module are then used as inputs into a Monte Carlo simulation model of the newsvendor problem. MCS is then used to evaluate different scenarios to determine the optimal advertising intensity and order quantity that maximizes the expected profit. The general structure of the proposed framework is provided in Figure 1. The following subsections provide detailed description of the ABMS and MCS modules of the framework.

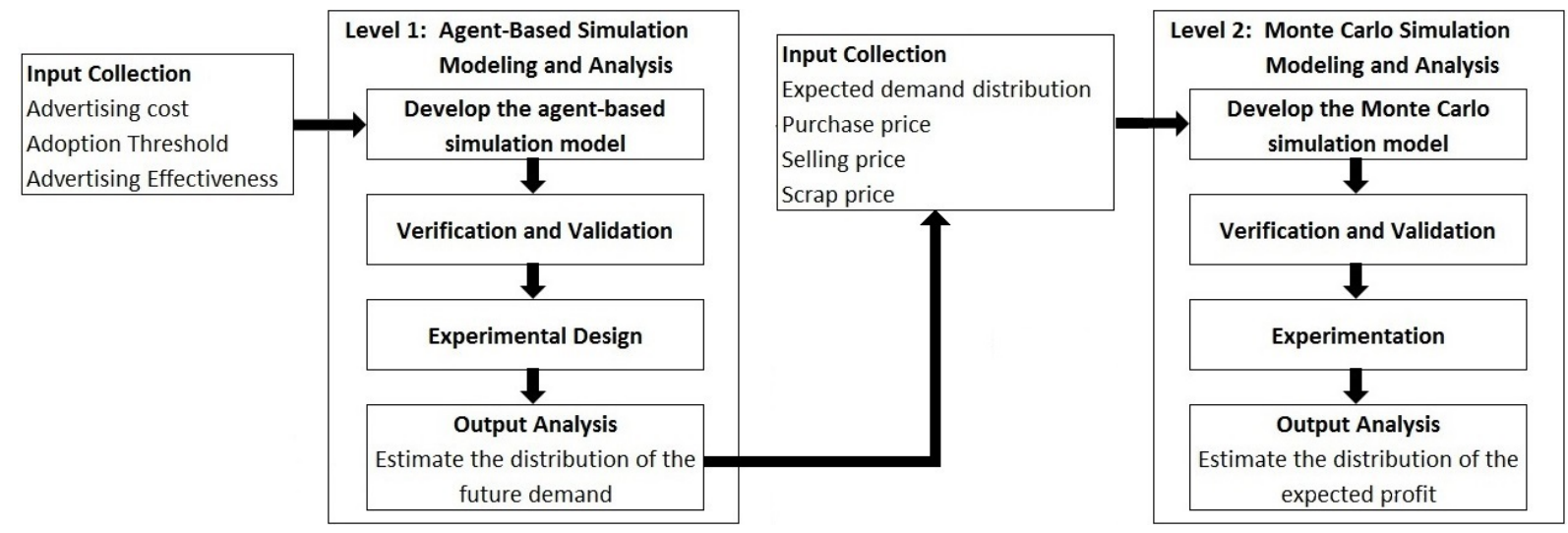

Figure 1: The general structure of the proposed two-level framework.

\subsection{Agent-Based Simulation Model}

Traditionally, simulation techniques have been widely applied in different fields ranging from manufacturing (Negahban and Smith 2013), healthcare (Mielczarek and Uzialko-Mydlikowska 2012), supply chain (Terzi and Cavalieri 2004), and military (Naseer, Eldabi, and Jahangirian 2009), to name a few. During the past two decades, with the advent of agent-based simulation, researchers have shown considerable interest in the application of simulation in social sciences (Axelrod 1997). Among all different areas within social sciences, consumer market place has become a major application area for this simulation technique. For a comprehensive analysis of the application of agent-based modeling and simulation to marketing research and practice, see Negahban and Yilmaz (2012). Agent-based simulation in marketing context generally involves building a model as a simplified representation of the market place where the primary unit of study is the autonomous consumer agent that interacts with other agents as well as the environment and makes decisions based on its behavioral rules. Therefore, agent-based models are bottom-up, i.e., macro-level phenomena emerge from these micro-level behaviors (Schelling 1978).

In the current paper, the market place under consideration consists of a number of consumers that reside in a grid-shaped environment and interact with each other through word-of-mouth and make decisions regarding the adoption of a new generic product. Consumers are engaged in a one-time purchase decision and the price of the product does not impact consumer buying decision since there is a single firm that sells the product (i.e., there is no competition in the market). The firm employs a direct advertising strategy to trigger the diffusion process and then relies on viral marketing to further penetrate into the market. Therefore, the proposed agent-based model takes into account both external (i.e., direct and mass media advertising) and internal (i.e., word-of-mouth) influences. This section provides a detailed description of the general agent-based simulation algorithm, consumers' decision making process, word-of-mouth operation, and the introductory advertising. 


\section{Negahban}

\subsubsection{Agent-Based Simulation Algorithm}

Consumers are divided into two general groups: undecided and adopters. The logic of the simulation process can be summarized as follows. The simulation begins by creating a virtual population of undecided consumers in a gird. At the beginning of the simulation run (i.e., at time zero), based on the intensity and effectiveness of the advertising, a number of undecided consumers adopt the new product. From this point on, the diffusion takes place under the word-of-mouth operation. At each time period, undecided consumers are influenced by their neighbors and make decision as to adopt or remain undecided. The distribution of the demand is then determined by replicating the simulation process and collecting data on the number of adopters during the peak sales period.

\subsubsection{Consumer Decision Making}

Rooted in different fields of sciences (e.g., behavioral and social sciences, psychology, economics, and marketing) consumer decision making has been found as one of the key elements of agent-based models of markets. Several approaches have been proposed to model the decision making process based on static or dynamic behavioral rules. Negahban and Yilmaz (2012) identify six main categories of decision making processes used in the literature as: (1) Personal preference; (2) State-based/stage-based; (3) Utility-based; (4) Exposure threshold; (5) Past experience; and (6) Random decision making. The reader is referred to the original article for detailed description of these categories and their applications.

Here, a variant of the threshold model is adopted. Threshold models (Granovetter 1978) characterize the social value of the product as being directly related to the proportion of adopters in the individuals neighborhood and thus represent the diffusion as a contagion process. Each individual has a personal threshold and when the number of adopters in her network of peers is greater than her personal threshold, she will adopt the product. Despite its simplicity, threshold models have shown to yield substantial results and thus have been widely applied in studying the diffusion of innovations, medical technologies, regulations and policies, etc. A comprehensive review of such applications is provided in Rogers (2003).

In this model, each potential consumer $i$ has a threshold for social pressure from its neighbors $\left(U_{i, \min }\right)$. When neighbors adopt the product, the normative influence on consumer $i$ in favor of the product increases. If the social influence for adoption, $x_{i, t}$, exceeds the minimum social threshold, $U_{i, \min }$, the individual will adopt the product. The social influence that consumer $i$ observes at time $t, x_{i, t}$, is determined by:

$$
x_{i, t}=\frac{\text { number of adopter neighbors at time } t}{\text { total number of neighbors }} .
$$

In this Model, we assume that the utility threshold for individual agents is uniformly distributed between 0 and 0.6, i.e. $U_{i, \min } \sim \mathrm{U}(0,0.6)$. As discussed in the literature (van Eck, Jager, and Leeflang 2011, Delre, Jager, and Janssen 2007), the choice of the uniform distribution serves for the purpose of introducing population heterogeneity into the model. Neighbors in the model can represent friends, co-workers, or any other type of relation that contributes to the social influence through word-of-mouth. In order to determine the neighbors of each consumer agent in the gird, the Moore neighborhood with a range of $r=2$ is used (Figure 2(a)). Therefore, in the proposed model, each individual has 24 neighbors, which is inline with the results provided by Goldenberg et al. (2007), who conduct a survey to estimate the number of ties and find that it varies from 3 to 28 and 1 to 40 for strong and weak ties, respectively.

Despite its simple structure, the literature provides various examples of the applicability of the Moore neighborhood (i.e., regular lattice network) and the significance of the insights derived from its application in social sciences. Schelling model of urban segregation based on preferences (Schelling 1978) provides a good example of such models that has made a significant contribution to the field. It is worth noting that as the significant effect of social system structure on the operation of word-of-mouth became better known (Rahmandad and Sterman 2008) and with the advancement of ABMS, the use of more complicated social network structures which overcome some of the shortcomings and simplifications of the Moore neighborhood has become prevalent in the state-of-the-art literature. These structures include small-world 


\section{Negahban}

networks (Watts and Strogatz 1998), preferential attachment (Barabási and Albert 1999), and empirical real-world networks based on social networking websites such as Twitter (Stonedahl, Rand, and Wilensky 2010). In this paper, for simplicity purposes, these network structures are not considered since the focus here is more on the methodological aspects of the integration of ABMS and MCS to solve NVP rather than proposing a complex agent-based model.

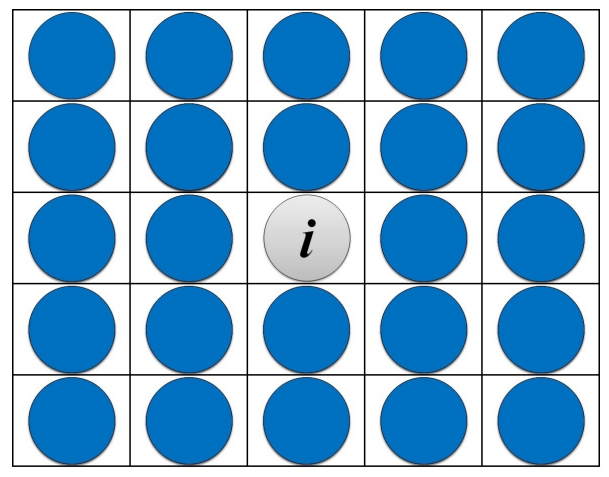

(a) Moore neighborhood with a range of $r=2$

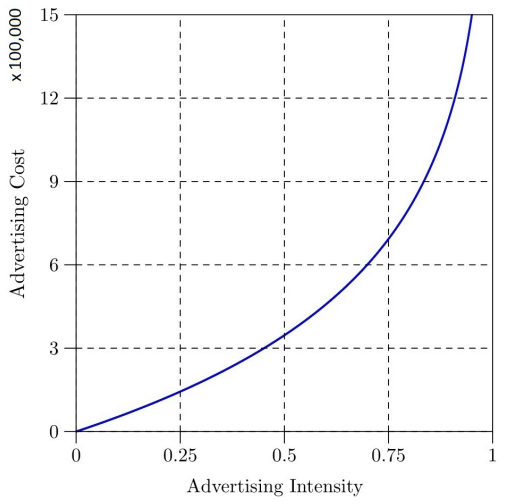

(b) The advertising cost function

Figure 2: Different components of the agent-based model.

As for the total number of consumers in the virtual market, it is found that that 1000 is sufficiently large to capture the underlying dynamics of the market and provide reliable results (Cowan and Jonard 2004, Alkemade and Castaldi 2005). Previous experimental design studies on larger networks also report that there is no significant difference in the simulation results obtained from markets of size 3000 and above (Goldenberg et al. 2007, Amini et al. 2012). In the current paper, in order to be more confident on the robustness of the simulation results, we model a virtual market consisting of 3600 consumers.

\subsubsection{Advertising Strategy}

In this model, individual consumers become aware of the product through introductory advertising. An individual who receives advertising will be convinced to adopt the product with a probability $f$, also known as advertising effectiveness. Advertising is costly. Let $C(p)$ denote the advertising cost of informing a proportion $p$ of consumers. According to the literature on advertising (Robert and Dale 1993; Orzach, Overgaard, and Tauman 2002), $C(\cdot)$ has to be continuously differentiable, strictly convex and increasing where $C(0)=0$ and $C(1)=\infty$. These characteristics can be justified as follows. An increasing convex function guarantees that it is increasingly costly to reach an extra proportion of the population. The condition $C(0)=0$ ensures that not advertising is costless and that nobody will become informed of the product if no advertising is done. On the other hand, $C(1)=\infty$ suggests that informing all individuals is extremely costly and thus infeasible. This will ensure that the optimal advertising intensity will always be less than 1. Based on the above, we consider an advertising cost function that satisfies all these conditions as follows:

$$
C(p)=\alpha \times \ln \left(-\frac{1}{p-1}\right),
$$

where $\alpha$ is a constant. Figure 2(b) illustrates the cost of advertising as a function of advertising intensity when $\alpha=5$.

\subsection{Monte Carlo Simulation Model}

In the second level of the proposed framework, a Monte Carlo simulation model of the newsvendor problem is developed to characterize the expected profit. The inputs into the MC model include the demand 


\section{Negahban}

distribution (as obtained from the ABMS module), selling price during the peak demand and clearance periods, unit purchase price, number of units ordered, and finally the initial advertising cost. We consider any combination of advertising intensity and order quantity as a scenario. For each scenario, random numbers are drawn from the distributions of the demand as well as peak selling and clearance prices. These sampled random variates are then used to calculate the total profit for a single run. The model is then replicated and simulation results are collected to obtain the expected profit for the current scenario. This process is repeated for all scenarios and the resulting expected profits are then compared to make the final decision on the advertising intensity and order quantity. The general structure of the Monte Carlo simulation algorithm can be summarized as follows in a pseudo-code format.

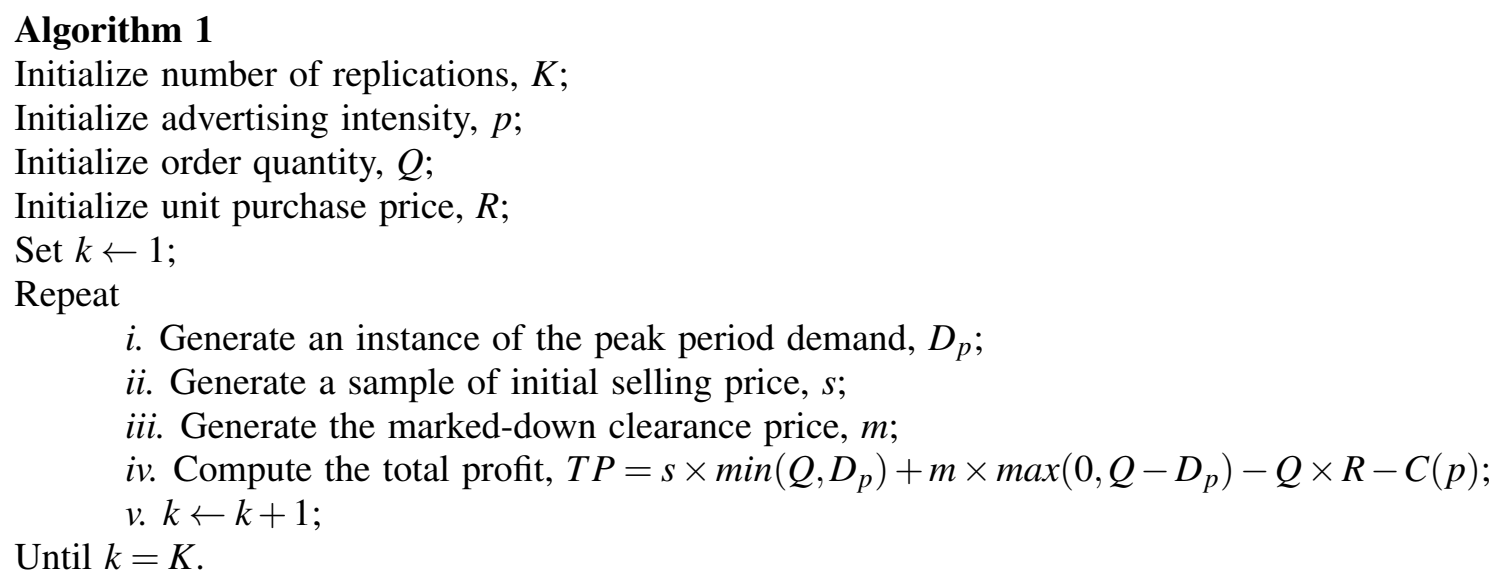

In step $i$ of the algorithm, the demand is denoted by $D_{p}$ and is sampled from the demand distribution associated with the chosen level of advertising intensity, $p$, as obtained from the ABMS analysis. Step $i v$. of the algorithm involves the calculation of total profit, where $s \times \min \left(Q, D_{p}\right)$ is the initial sales during the peak demand period. If the peak period demand, $D_{p}$, is greater than the order quantity, $Q$, then there will be no remaining inventory to be sold as clearance. Therefore, the sales during the clearance period is calculated by $m \times \max \left(0, Q-D_{p}\right)$. Moreover, the total purchase cost is $Q \times R$ and the cost associated with the initial advertising efforts is $C(p)$, which is calculated by (2).

\section{FRAMEWORK IMPLEMENTATION}

This section illustrates the application of the proposed framework to a hypothetical newsvendor problem. Experimental design, verification and validation of the two simulation models, and the results are discussed.

\subsection{A Hypothetical Newsvendor Problem}

The newsvendor problem presented here is adopted from (Seila, Ceric, and Tadikamalla 2003) with a few modifications. Consider a large merchandise catalog company that is planning to have a special furniture sale a month from now. The company must place an order for the new type of furniture at this time. The supplier that sells the furnitures offers four different contract options as follows:

1. 500 chairs at a cost of $\$ 200$ per unit;

2. 1000 chairs at a cost of $\$ 190$ per unit;

3. 1500 chairs at a cost of $\$ 175$ per unit; and

4. 2000 chairs at a cots of $\$ 165$ per unit.

The merchandise company plans to sell each unit of the product at a price of $s$ during the initial sales period which is going to last for 10 days. After this period, it is assumed that all remaining units will be 


\section{Negahban}

sold during a clearance period at a price of $m$ which is half of the original price (i.e., $m=s / 2$ ). While the order quantity and the unit purchase cost are set contractually, the number of units sold during the first 10 days and the initial selling price are random variables that depend on the dynamics of the market. Pricing analysis indicates that the initial price will be between $\$ 230$ and $\$ 340$, i.e. $s \sim U(230,340)$. The company employs a combination of different direct advertising and viral marketing to boost its sales. The cost associated with informing a proportion $p$ of the potential consumer population is estimated to follow the expression provided in (2) with $\alpha=5$ (see Figure 2(b)). Based on historical data, the effectiveness of direct advertising is estimated to be $5 \%$. The company would like to evaluate the performance of different combinations of advertising intensity and contract option in terms of expected profit.

\subsection{Experimental Design}

Based on the above, the selected parameter setting for implementation of the proposed framework is summarized in Table 1. There are two factors in the experimental design that are considered as independent design factors, namely contract option and advertising intensity, $p$. There are four contract options. Here, we vary the level of advertising intensity from 5\% to 30\% in inceremnts of 5\% (i.e., 6 levels). An analysis on the half-width values for the results from several pilot runs indicates that the minimum number of replications necessary for the agent-based simulation model to identify the demand distribution is 300 . Therefore, a total of $6 \times 300=1800$ agent-based simulation runs are performed. In order to evaluate different combinations of advertising intensity and contract option, we run the Monte Carlo simulation model for a total of $4 \times 6=24$ scenarios, each replicated for 5000 iterations to be confident in the accuracy of the results. Thus, $24 \times 5000=120,000$ Monte Carlo simulation runs are performed. The agent-based model is developed in Repast Simphony (North et al. 2013), which is a Java-based agent-oriented simulation platform, and the Monte Carlo model is developed in Matlab ${ }^{\circledR}$. Simulation experiments for both models are run on a standard Dell ${ }^{\mathrm{TM}}$ desktop with a $3.00 \mathrm{GHz}$ quad-core CPU and 8GB of RAM.

Table 1: Parameter choices for the agent-based and Monte Carlo simulation models.

\begin{tabular}{lll}
\hline Model & Parameter & Value/Range \\
\hline Agent-based model & Population size & 3600 \\
& Social pressure threshold $\left(U_{i, \min }\right)$ & $U(0,0.6)$ \\
& Type of neighborhood & Moore's neighborhood \\
& Number of neighbors & 24 \\
& Advertising intensity $(p)$ & $5 \%-30 \%$ \\
& Advertising effectiveness & $5 \%$ \\
& Number of replications & 300 \\
Monte Carlo model & Order quantity $(Q)$ & $500,1000,1500,2000$ \\
& Unit purchase price/cost $(R)$ & $200,190,175,165$ \\
& Initial selling price $(s)$ & $U(230,340)$ \\
& Clearance selling price $(m)$ & $s / 2$ \\
& Advertising cost $(C(p))$ & As determined by $(2)$ \\
& Number of replications $(K)$ & 5000 \\
\hline
\end{tabular}

\subsection{Verification and Validation}

Before conducting the simulation experiments, verification and validation are performed according to the guidelines available in the literature (Sargent 2005; Rand and Rust 2011). Verification and validation steps performed to gain confidence in the correctness of the models can be summarized as follows. Both simulation models are tested to ensure that they are error free and properly implemented. Using structured walk-through and trace techniques, different simulation functions such as time-flow and pseudo-random 


\section{Negahban}

number generators are tested to be correctly implemented. Once the models are verified, a combination of different techniques are employed to validate the agent-based model. Animation is used to graphically monitor the behavior of individuals in the population. Degenerate tests and extreme condition tests are then performed to observe the behavior of the model. For example, when advertising level is zero, the diffusion will not take off. Operational graphics are also used to dynamically display the output measures as the model is being run to ensure that they behave correctly. Finally, sensitivity analysis on two main model parameters is conducted to determine that the model behaves as expected when these parameters vary. The sensitivity analysis can be described as follows:

- Given all other parameters remain unchanged, an increase in advertising effectiveness will expedite the diffusion process and thus increase the total number of adopters during the initial sales period. We vary the advertising effectiveness from 0.01 to 0.06 to observe its impact on the initial sales which matches our expectations (Figure 3(a)).

- Smaller social pressure threshold, $U_{i, \min }$, is expected to increase the initial sales since consumers will be more likely to adopt the product when only a few neighbors have already adopted. Under a given advertising intensity of $10 \%$ with an effectiveness of 0.05 , this hypothesis is tested and verified by changing the upper bound for $U_{i, \min }$ from 0.1 to 0.9 (Figure 3(b)).

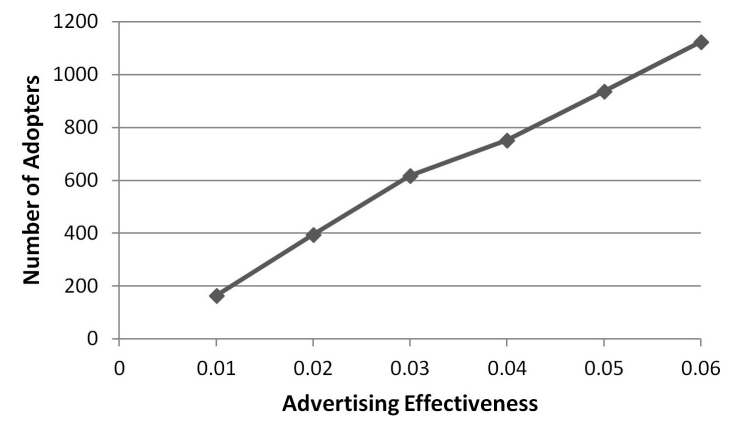

(a)

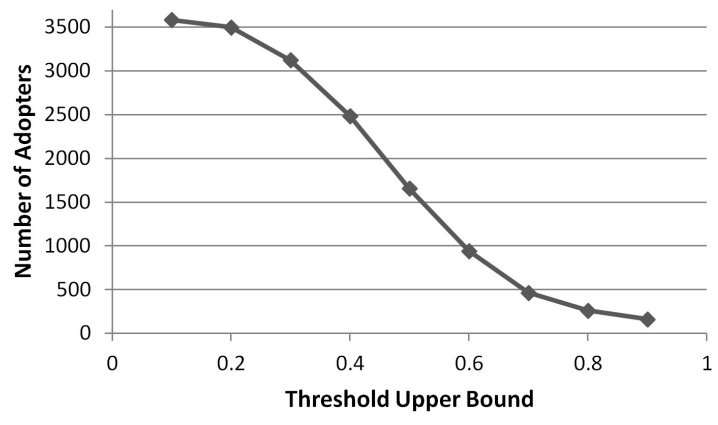

(b)

Figure 3: Results of the validation runs.

\subsection{Analysis of Simulation Results}

Once the agent-based simulation model is developed, verified and validated, it is used to predict the distribution of the peak period demand under different levels of initial advertising intensity. Figure 4 illustrates the histograms for the peak period demand obtained from the ABMS analysis. Before using these results in the Monte Carlo simulation model, the histograms are approximated by fitting an appropriate probability distribution to the corresponding data set. For this purpose, we used Minitab ${ }^{\circledR}$ and EasyFit ${ }^{\circledR}$ software packages to evaluate the fitness of four commonly used distributions namely normal, lognormal, weibull, and gamma distributions. The results of the goodness-of-fit test are summarized in Table 2, which suggests that the normal distribution provides the best fit for the distribution of demand for the cases of advertising intensities of $5 \%, 10 \%, 20 \%$, and $25 \%$, while the Weibull distribution reports the best fit for the remaining two scenarios (i.e., $15 \%$ and $30 \%$ as the initial advertising intensity). Moreover, as expected, average demand during the peak period increases as advertising intensity is increased.

Based on the predictions of the demand from the agent-based model, Monte Carlo simulation is employed to compare the expected total profit for the 24 possible scenarios. Figure 5(a) depicts the impact of different levels of initial advertising intensity on the profit for each contract option. The results indicate that an advertising intensity of $20 \%$ along with the choice of the $4^{\text {th }}$ contract option maximizes the expected 


\section{Negahban}

total profit of the merchandise company. Despite the simplicity of the problem, two important insights can be derived from the results which further indicates the significance of such analysis. The first interesting observation is that restrictions on the level of advertising, for example due to limited resources such as advertising budget, can change the choice of the contract. In other words, it is not always the case that the same contract option performs best for all advertising levels. For the newsvendor problem under consideration, for the 5\% level of initial advertising, contract number 1 gives the highest expected profit while the $4^{\text {th }}$ contract maximizes the profit for the rest of advertising intensity levels.

Secondly, based on the assumptions of the threshold model used in this paper, initial adoption only takes place as a result of advertising, i.e., the product will not take off if the introductory advertising fails. It is obvious that a higher advertising intensity would increase the diffusion speed and thus sales during the peak period. However, a higher advertising intensity does not necessarily yield more profit due to the special structure of the advertising cost function which brings interesting trade-offs into the problem. The observation that the plot for each contract option peaks at a different level of advertising indicates that the optimal advertising level varies based on the order quantity and supplier's discounting policy.

Perhaps the most important advantage of the proposed approach is that it allows for risk-based analysis. This is not possible if analytical methods are used since they typically report only the expected value. Figure 5(b) provides the cumulative distribution function (CDF) plot of the optimal scenario (i.e., contract 4 and advertising level of 20\%). Although an expected profit of $\$ 81,820$ is reported for this scenario, according to the figure, there is about a $15 \%$ probability that the firm will still lose money. If this amount of risk is beyond the company's tolerance, the managers may decide to choose a more conservative option with a lower expected profit to minimize the probability of losing money. On the other hand, there is a $17 \%$ chance that the company will make more than $\$ 150,000$ in profits if they decide to go with this scenario. Therefore, in making the final decision, there is typically a trade-off between the expected profit and risk. The final decision in the newsvendor problem should be made based on a comprehensive risk analysis rather than the mere value of the expected profit.

\section{CONCLUSIONS}

In this paper, an overview of the literature on the newsvendor problem is provided and existing gaps are identified. To address the deficiencies in the literature, a novel hybrid approach based on agent-based and Monte Carlo simulation is proposed. The main advantage of the proposed tool lies in its ability to analyze the impact of advertising, supplier discounting policies, word-of-mouth and viral marketing at the same time, which has not been previously addressed in the literature. The applicability of the proposed framework is illustrated through an example of a single-period newsvendor problem. The results from the two simulation models are then analyzed in order to determine the optimal order quantity and initial advertising level in the presence of viral marketing with the objective to maximize the expected profit.

It is worth noting that the goal of this paper is to provide a groundwork for the integration of agentbased modeling and Monte Carlo simulation techniques to solve the newsvendor problem. The proposed agent-based model involves several simplifying assumptions and certainly does not capture all dimensions of advertising, word-of-mouth, social network structure, or consumer decision making process. Moreover, many practitioners may find these assumptions unrealistic. Therefore, the validity of the agent-based model is an important aspect that requires more attention in the future through the incorporation of different theoretical or empirical network structures and more realistic and complex behavioral rules based on recent advancements in psychological theories on consumer behavior. The application of the ABMS-MCS approach to multi-period and multi-product newsvendor problem is another interesting area for future research. Finally, although briefly discussed in this study, simulation is a powerful tool for characterizing the risk associated with different decision choices. Considering the firm's risk profile in the final decision on the economical order quantity could also be addressed in future studies. The authors strongly believe that addressing the above issues would increase the credibility of the proposed approach and its adoption by researchers and practitioners as a decision support tool in real-world newsvendor-like settings. 
Negahban

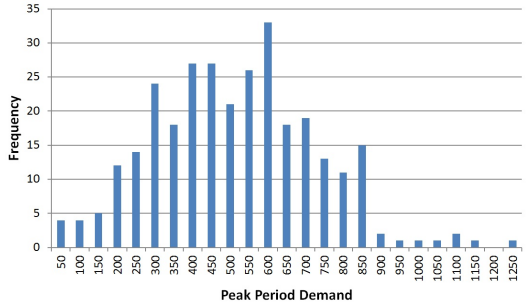

(a) Advertising intensity $=5 \%$

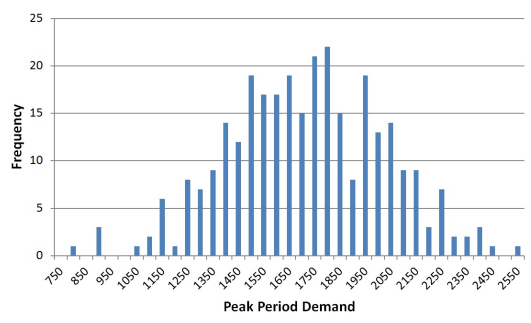

(d) Advertising intensity $=20 \%$

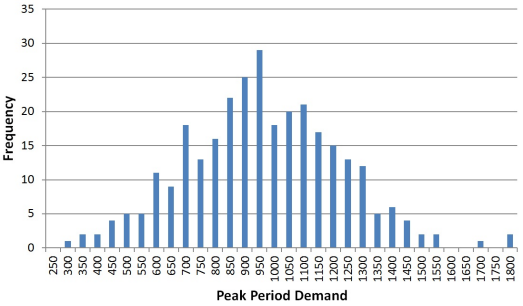

(b) Advertising intensity $=10 \%$

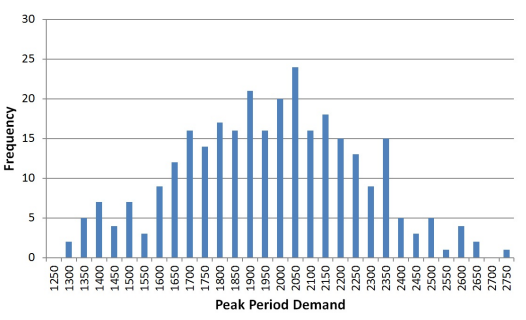

(e) Advertising intensity $=25 \%$

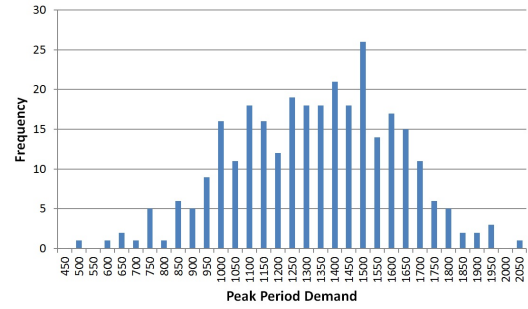

(c) Advertising intensity $=15 \%$

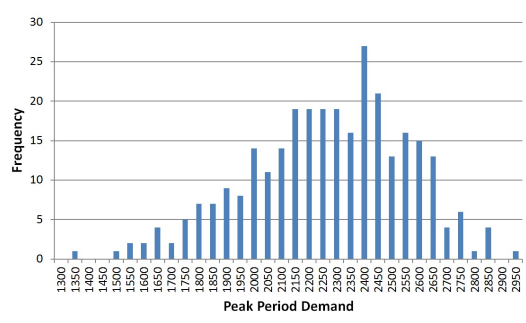

(f) Advertising intensity $=30 \%$

Figure 4: Histograms of peak demand distribution obtained from the ABMS module.

Table 2: Results of the goodness-of-fit test.

\begin{tabular}{|c|c|c|c|}
\hline Advertising Intensity & Distribution & $P$-value & Best Fit \\
\hline \multirow[t]{4}{*}{$5 \%$} & Normal(488.47,211.14) & 0.333 & $\checkmark$ \\
\hline & Lognormal $(6.05,0.62)$ & $<0.005$ & \\
\hline & Weibull(1.89,575.88,0.00) & $>0.25$ & \\
\hline & $\operatorname{Gamma}(5.35,91.26)$ & $<0.005$ & \\
\hline \multirow[t]{4}{*}{$10 \%$} & Normal(938.36,261.97) & 0.957 & $\checkmark$ \\
\hline & Lognormal $(6.80,0.30)$ & $<0.005$ & \\
\hline & Weibull(4.09,1030.4,0.00) & $>0.25$ & \\
\hline & $\operatorname{Gamma}(12.83,73.13)$ & $<0.005$ & \\
\hline \multirow[t]{4}{*}{$15 \%$} & Normal(1306.1,281.54) & 0.086 & \\
\hline & Lognormal $(7.14,0.23)$ & $<0.005$ & \\
\hline & Weibull(5.35,1413.8,0.00) & 0.913 & $\checkmark$ \\
\hline & $\operatorname{Gamma}(21.52,60.68)$ & $<0.005$ & \\
\hline \multirow[t]{4}{*}{$20 \%$} & Normal(1691.5,308.63) & 0.913 & $\checkmark$ \\
\hline & Lognormal(7.41,0.19) & 0.006 & \\
\hline & Weibull $(6.51,1811,0.00)$ & 0.038 & \\
\hline & $\operatorname{Gamma}(30.03,56.31)$ & 0.164 & \\
\hline \multirow[t]{4}{*}{$25 \%$} & Normal(1940.5,294.14) & 0.769 & $\checkmark$ \\
\hline & Lognormal(7.55,0.15) & $<0.005$ & \\
\hline & Weibull $(3.43,1006.7,1036.1)$ & 0.068 & \\
\hline & $\operatorname{Gamma}(43.52,44.58)$ & 0.043 & \\
\hline \multirow[t]{4}{*}{$30 \%$} & Normal $(2243,288.7)$ & 0.013 & \\
\hline & Lognormal(7.70,0.13) & $<0.005$ & \\
\hline & Weibull(9.31,2361.6,0.00) & 0.909 & $\checkmark$ \\
\hline & $\operatorname{Gamma}(60.36,37.15)$ & $<0.005$ & \\
\hline
\end{tabular}




\section{Negahban}

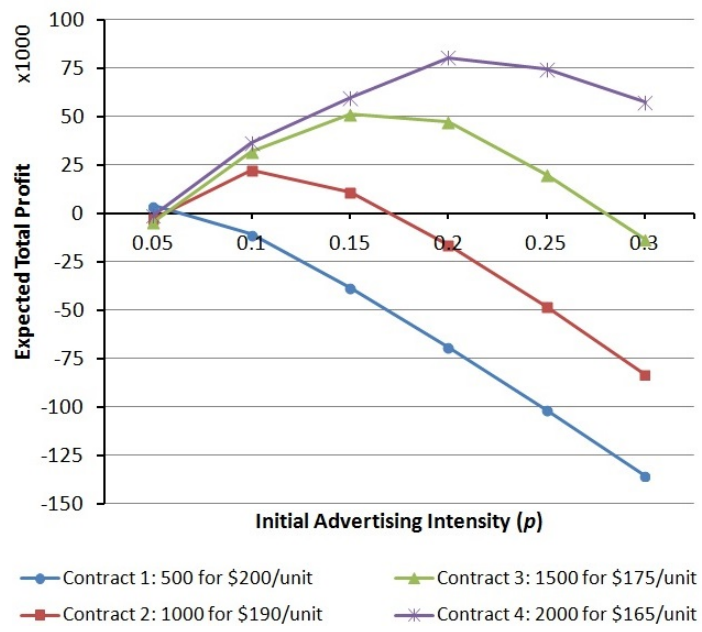

(a) Expected total profit for each scenario

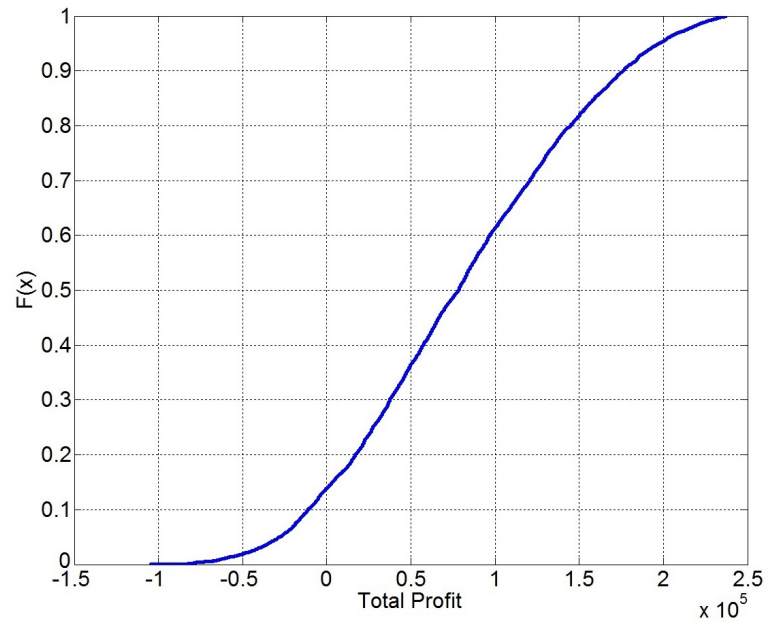

(b) The CDF plot of profit for the optimal scenario

Figure 5: Monte Carlo simulation outputs.

\section{REFERENCES}

Alkemade, F., and C. Castaldi. 2005. "Strategies for the Diffusion of Innovations on Social Networks". Computational Economics 25:3-23.

Amini, M., T. Wakolbinger, M. Racer, and M. G. Nejad. 2012. "Alternative Supply Chain Production-Sales Policies for New Product Diffusion: An Agent-Based Modeling and Simulation Approach". European Journal of Operational Research 216 (2): 301-311.

Axelrod, R. 1997. "Advancing the Art of Simulation in the Social Sciences". Complexity 3 (2): 16-22.

Barabási, A.-L., and R. Albert. 1999. "Emergence of Scaling in Random Networks". Science 286:509-512.

Cowan, R., and N. Jonard. 2004. "Network Structure and the Diffusion of Knowledge". Journal of Economic Dynamics and Control 28 (8): 1557-1575.

Delre, S., W. Jager, and M. Janssen. 2007. "Diffusion Dynamics in Small-world Networks with Heterogeneous Consumers". Computational \& Mathematical Organization Theory 13:185-202.

Eeckhoudt, L., C. Gollier, and H. Schlesinger. 1995. "The Risk-Averse (and Prudent) Newsboy". Management Science 41 (5): 786-794.

Eynan, A., and M. J. Rosenblatt. 1995. "Assemble to Order and Assemble in Advance in a Single-period Stochastic Environment”. Naval Research Logistics (NRL) 42 (5): 861-872.

Goldenberg, J., B. Libai, S. Moldovan, and E. Muller. 2007. "The NPV of Bad News". International Journal of Research in Marketing 24 (3): 186-200.

Granovetter, M. 1978. "Threshold Models of Collective Behavior". American Journal of Sociology 83 (6): $1420-1443$.

Jucker, J. V., and M. J. Rosenblatt. 1985. "Single-period Inventory Models with Demand Uncertainty and Quantity Discounts: Behavioral Implications and a New Solution Procedure". Naval Research Logistics Quarterly 32 (4): 537-550.

Khouja, M. 1999. "The Single-period (News-vendor) Problem: Literature Review and Suggestions for Future Research". Omega 27 (5): 537-553.

Kraiselburd, S., V. G. Narayanan, and A. Raman. 2004. "Contracting in a Supply Chain with Stochastic Demand and Substitute Products". Production and Operations Management 13 (1): 46-62.

Lau, H.-S. 1980. "The Newsboy Problem under Alternative Optimization Objectives". The Journal of the Operational Research Society 31 (6): 525-535.

Mielczarek, B., and J. Uzialko-Mydlikowska. 2012. "Application of Computer Simulation Modeling in the Health Care Sector: A Survey”. SIMULATION 88 (2): 197-216. 


\section{Negahban}

Murray, G. R., and E. A. Silver. 1966. "A Bayesian Analysis of the Style Goods Inventory Problem". Management Science 12 (11): 785-797.

Nahmias, S., and C. P. Schmidt. 1984. "An Efficient Heuristic for the Multi-item Newsboy Problem with a Single Constraint". Naval Research Logistics Quarterly 31 (3): 463-474.

Naseer, A., T. Eldabi, and M. Jahangirian. 2009. "Cross-sector Analysis of Simulation Methods: A Survey of Defense and Healthcare". Transforming Government: People, Process and Policy 3 (2): 181-189.

Negahban, A., and J. S. Smith. 2013. "Simulation for Manufacturing System Design and Operation: A Comprehensive Survey". Working paper.

Negahban, A., and L. Yilmaz. 2012. "Agent-Based Simulation Applications in Marketing Research: An Integrated Review". Submitted to the Journal of Simulation (under review).

North, M., N. Collier, J. Ozik, E. Tatara, C. Macal, M. Bragen, and P. Sydelko. 2013. "Complex Adaptive Systems Modeling with Repast Simphony". Complex Adaptive Systems Modeling 1 (1): 3.

Orzach, R., P. B. Overgaard, and Y. Tauman. 2002. "Modest Advertising Signals Strength". The RAND Journal of Economics 33 (2): 340-358.

Petruzzi, N. C., and M. Dada. 1999. "Pricing and the Newsvendor Problem: A Review with Extensions". Operations Research 47 (2): 183-194.

Qin, Y., R. Wang, A. J. Vakharia, Y. Chen, and M. M. Seref. 2011. "The Newsvendor Problem: Review and Directions for Future Research”. European Journal of Operational Research 213 (2): 361-374.

Rahmandad, H., and J. Sterman. 2008. "Heterogeneity and Network Structure in the Dynamics of Diffusion: Comparing Agent-Based and Differential Equation Models". Management Science 54 (5): 998-1014.

Rand, W., and R. T. Rust. 2011. "Agent-Based Modeling in Marketing: Guidelines for Rigor". International Journal of Research in Marketing 28 (3): 181-193.

Robert, J., and O. S. Dale. 1993. "Informative Price Advertising in a Sequential Search Model". Econometrica 61 (3): 657-686.

Rogers, E. M. 2003. Diffusion of Innovations. 5th ed. New York, NY, USA: Free Press.

Sargent, R. G. 2005. "Verification and Validation of Simulation Models". In Proceedings of the 2005 Winter simulation Conference, edited by M. E. Kuhl, N. M. Steiger, F. B. Armstrong, and J. A. Joines, 130-143. Institute of Electrical and Electronics Engineers, Inc.

Schelling, T. C. 1978. Micromotives and Macrobehavior. New York, NY, USA: W.W. Norton.

Seila, A., V. Ceric, and P. Tadikamalla. 2003. Applied Simulation Modeling. Belmont, CA, USA: ThomsonBrooks/Cole (Duxbury).

Stonedahl, F., W. Rand, and U. Wilensky. 2010. "Evolving Viral Marketing Strategies". In Proceedings of the 12th Annual Conference on Genetic and Evolutionary Computation, 1195-1202.

Terzi, S., and S. Cavalieri. 2004. "Simulation in the Supply Chain Context: A Survey". Computers in Industry 53 (1): 3-16.

Urban, T. L. 2002. "The Interdependence of Inventory Management and Retail Shelf Management". International Journal of Physical Distribution \& Logistics Management 32 (1): 41-58.

van Eck, P. S., W. Jager, and P. S. H. Leeflang. 2011. "Opinion Leaders' Role in Innovation Diffusion: A Simulation Study”. Journal of Product Innovation Management 28 (2): 187-203.

Watts, D. J., and S. H. Strogatz. 1998. "Collective Dynamics of 'Small-world'Networks". Nature 393 (6684): 440-442.

\section{AUTHOR BIOGRAPHY}

ASHKAN NEGAHBAN is an instructor and PhD student at the Department of Industrial and Systems Engineering at Auburn University. He received his Master's and BSc both in Industrial Engineering from Auburn University and University of Tehran, respectively. He teaches courses in simulation modeling and analysis at the undergraduate level. His research interest is in development of simulation-based methodologies with a specific application to manufacturing systems. His email address is anegahban@auburn.edu. 